\title{
Metabolic Syndrome and Cardiovascular Risk in Liver Transplant Recipients - Can Non-invasive Testing Change the Landscape?
}

\author{
Carmen Ester ${ }^{1,2}$, Razvan Cerban ${ }^{1,2}$, Speranta lacob ${ }^{1,2}$, Corina Pietrareanu $^{1}$, Mihaela Lita ${ }^{1}$, \\ Olteanu Ovidiu-Andrei ${ }^{1}$, Simona Ichim ${ }^{1}$, Georgiana Constantin ${ }^{3}$, Liliana Paslaru ${ }^{2,3}$, Camelia Grancea ${ }^{4}$, \\ Simona Ruta ${ }^{2,4}$, Liana Gheorghe ${ }^{1,2}$
}

\author{
Corresponding author: \\ Liana Gheorghe, MD, PhD \\ Department of Gastroenterology \\ and Hepatology, Fundeni Clinica \\ Institute, Bucharest, Romania \\ E-mail: drlgheorghe@gmail.com
}

\section{Abbreviations:}

MetS - metabolic syndrome

NAFLD - on-alcoholic fatty liver disease

TE - transient elastography

$\mathrm{BMI}$ - body mass index

CAP - controlled attenuation parameter
'Department of Hepatology and Liver Transplantation, Fundeni Clinical Institute, Bucharest, Romania

${ }^{2}$ Carol Davila University of Medicine and Pharmacy, Bucharest, Romania

${ }^{3}$ Department of Biochemistry - Liver Transplant Unit, Fundeni Clinical Institute, Bucharest,

Romania

${ }^{4}$ Stefan S Nicolau Institute of Virology, Bucharest, Romania

\section{ABSTRACT}

Objective: Liver transplant recipients often develop metabolic syndrome (MetS) and de-novo non-alcoholic fatty liver disease (NAFLD). Our aim was to evaluate the cardiovascular risk in these patients using CXCL10,a serum biomarker whose expression levels have been associated with inflammatory diseases.

Methods: We assessed 60 liver transplant recipients for clinical and biological features. The cardiovascular risk was assessed using the Framingham risk score.

Results: HCV cirrhosis was the main indication for liver transplantation (69\% of patients) and the mean age 56 years. The paired t-test showed a significant association of CXCL-10 with the presence of the metabolic syndrome $(p<0,001)$ and also with the non-invasive scores for advanced fibrosis and NAFLD. The metabolic syndrome was present in $51 \%$ of patients. $23 \%$ of patients had a Framingham risk score higher than 12 (10\% mortality in 10 years) and $13 \%$ a score higher than 17 (30\% mortality in 10 years). A significant association between the serum levels of CXCL10 and ferritin $(p=0,04)$, urea $(p=0,003)$, but also with uric acid $(p=0,04)$, an already established risk factor for cardiovascular mortality was shown in the Spearman rank correlation test showed. The multivariate analysis indicated as independent prediction factors for higher risk indicated by the Framingham score the serum levels of ferritin $(p=0,03)$ and urea $(p=0,02)$.

Conclusion: CXCL10 might be a useful tool in assessing the cardiovascular risk in liver transplant recipients, especially when features of the metabolic syndrome are present. Our study is ongoing, aiming to establish the role of biomarkers, including CXCL10 in cardiovascular related mortality in these patients.

Key words: non-alcoholic fatty liver disease, cardiovascular mortality, liver transplant recipients

\section{INTRODUCTION}

NAFLD is an expanding health problem and is predicted to become the most 
common cause of chronic liver disease in the world.

NAFLD is defined as the accumulation of lipids, especially triglycerides, in more than $5 \%$ of the hepatocytes, after excluding chronic ethanol consumption, as well as other related causes (1). Traditionally NAFLD has been considered a simple hepatic manifestation of the metabolic syndrome, but recent evidence point toward a mutual interrelationship between the evolution of NAFLD and the increased complications caused by type 2 diabetes or cardiovascular disease (2). Liver transplant recipients often develop metabolic syndrome and de-novo NAFLD, there is a large debate regarding NAFLD as a cause or a consequence of the metabolic syndrome and its individual components.

Cardiovascular disease is the leading cause of death in patients with NAFLD who present frequently central obesity, atherogenic dyslipidemia, and arterial hypertension.

The Framingham Heart Study investigators concluded that arterial hypertension and other components of the metabolic syndrome were all positively associated with an increased risk of incident NAFLD, independent of adiposity measures (3). A Swedish study with 229 patients with biopsy-proven NAFLD demonstrated that liver fibrosis stage was the strongest predictor for disease specific mortality after a long period of follow-up. In patients with NAFLD an increased risk of fatal and nonfatal cardiovascular complications was observed and attributed to subclinical myocardial remodelling and dysfunction, valvular disease (aortic valve sclerosis and mitral annular calcification) or arrhytmias (mostly atrial fibrillations and Qtc prolongation) (4).

A series of immune biomolecules have been studied as candidate biomarkers for diagnosis or prognosis of cardiovascular complications. CXCL-10, a small protein, member of the CXC chemokine family, that plays important roles in chemoattraction for monocytes/ macrophages, T cells, NK cells, dendritic cells, and promotion of $\mathrm{T}$ cell adhesion to endothelial cells has been studied in a series of inflammatory diseases (ref) and has been used in hepatitis $C$ treated patients to predict treatment response to interferon/ribavirin (5). Our aim was to assess the predictive value of CXCL10 for cardiovascular risk in patients with NAFLD.

\section{PATIENTS AND METHODS}

\section{Study population}

Sixty liver transplant recipients were assessed at 6-12 month after transplantation for clinical and biological features (liver enzymes, cholesterole, tryglicerides, fasting glucose, ferritin, urea, creatinine, $\mathrm{C}$-reactive protein. The medical history for diabetes, arterial hypertension and medication was recorded for each patient. The body mass index (BMI), waist circumference, tryglicerides, HDL-cholesterol, fasting glucose level and blood pressure were all incorporated into the International Diabetes Federation criteria to define metabolic syndrome.

Abdominal ultrasound and transient elastography (TE) with controlled attenuation parameter (CAP) were performed for all patients and, non-invasive scoring systems for advanced fibrosis (APRI, FIB-4) and NAFLD score were calculated. Many studies identified the optimal cutoff APRI value for significant fibrosis 0.456 , for FIB-4 > 3.25 (7) and NAFLD fibrosis score (NFS) > 0.676 is reported to be associated with about a fourfold increase in the risk of death as compared with anormal value and NFS is associated with higher risk of mortality in a dose-response manner (8). The cardiovascular risk was assessed using the Framingham risk score (9) and the presence of metabolic syndrome.

\section{Ultrasonography and elastography evaluation}

All patients performed abdominal ultrasound first, a degree of liver fat acuumulation was estimated using measurements of the difference of liver-kidney echo amplitude, visual determination of the echo penetration into the depth of the liver and the estimation of the clear visualization of the vessels inside the liver. The degree of liver fibrosis and steatosis was estimated using Fibroscan ${ }^{\circledR}$ with controlled attenuation parameter (CAP) module.

\section{Statistical analysis}

Statistical analysis was performed using MedCalc Statistical Software version 18.2.1 (MedCalc Software bvba, Ostend, Belgium; http://www.medcalc.org; 2018).

We report data as means and standard deviation and differences between the normally distributed patients characteristics were analysed using Student's t-test and matched pair samples were further seen in paired t-test. The Spearman rank correlation coefficient was used to assess the monotonic relationship between 2 variables. For the multivariate analysis we tested the following predictors for the Framingham risk score: ferritin, urea, creatinine, $\mathrm{C}$-reactive protein, $\mathrm{BMI}$ and the presence of diabetes mellitus. A p-value less than 0,05 was considered statistically significant. 


\section{RESULTS}

\section{Baseline characteristics}

Of the sixty liver transplant recipients $45 \%$ had F0-F1 grade fibrosis on Fibroscan ${ }^{\circledR}$, while the CAP measurement identified $53 \%$ of patients with important liver steatosis ( $\geq$ grade 2 ).

The frequency of type 2 diabetes mellitus in the studied population was 60\% (36 patients) of which 12 patients (20\%) had new onset type 2 diabetes mellitus after liver transplantation. Regarding the presence of arterial hypertension the situation was similar with 30 patients (50\%) being diagnosed with high blood pressure after liver transplantation, with a higher rate of new onset arterial hypertension of $32 \%$ (19 patients).

The Framingham risk score for cardiovascular mortality was calculated in all patients as a gender specific algorithm used to estimate the 10 years cardiovascular risk of an individual. In our study $13 \%$ of the patients were assessed to have a high cardiovascular risk at 10 years ( $2 \% /$ year).

The incidence of metabolic syndrome was 52\% (32 patients) in the studied population (table 1).

Table 1 - Summary statistics table

\begin{tabular}{|c|c|c|c|c|}
\hline & Mean & Median & SD & RSD \\
\hline Uric acid & 6.557 & 6.300 & 1.4540 & 0.2218 \\
\hline $\mathrm{ALT}$ & 72.407 & 34.000 & 80.3898 & 1.1103 \\
\hline APRI & 1.297 & 0.651 & 2.1758 & 1.6778 \\
\hline AST & 56.610 & 31.000 & 81.0332 & 1.4314 \\
\hline BMI & 26.789 & 26.590 & 4.4387 & 0.1657 \\
\hline Total cholesterol & 170.153 & 163.000 & 44.6966 & 0.2627 \\
\hline Creatinine & 1.071 & 0.950 & 0.4303 & 0.4017 \\
\hline CXCL10 & 266.817 & 186.000 & 223.6243 & 0.8381 \\
\hline Diabetes mellitus & 0.600 & 1.000 & 0.4940 & 0.8234 \\
\hline Ferritin & 175.616 & 106.000 & 213.8879 & 1.2179 \\
\hline FIB4 & 3.003 & 2.206 & 2.6505 & 0.8827 \\
\hline Framingham_risk_score & 7.630 & 4.550 & 7.6642 & 1.0045 \\
\hline Fibroscan & 9.022 & 5.800 & 8.0265 & 0.8897 \\
\hline CAP values & 235.455 & 259.000 & 87.3361 & 0.3709 \\
\hline $\begin{array}{l}\text { Presence of arterial } \\
\text { hypertension }\end{array}$ & 0.517 & 1.000 & 0.5041 & 0.9745 \\
\hline $\begin{array}{l}\text { Median time since } \\
\text { transplantation (days) }\end{array}$ & 1201.767 & 1069.500 & 676.6069 & 0.5630 \\
\hline $\begin{array}{l}\text { Presence of metabolic } \\
\text { syndrome }\end{array}$ & 0.533 & 1.000 & 0.5031 & 0.9433 \\
\hline NAFLD score & -2.088 & -2.001 & 0.8584 & -0.4111 \\
\hline C reactive protein & 6.180 & 3.980 & 7.0201 & 1.1360 \\
\hline Triglycerides & 136.759 & 119.500 & 72.6449 & 0.5312 \\
\hline Urea & 48.306 & 44.200 & 20.8397 & 0.4314 \\
\hline
\end{tabular}
tation was HCV infection (69\% of patients) and the mean age 56 years (fig. 1 ).

The paired t-test showed a significant association of CXCL-10 with the presence of the metabolic syndrome $(p<0,001)$ and also with the non-invasive scores for advanced fibrosis (APRI \& FIB4) and NAFLD (figs. 2, 3).

The metabolic syndrome was present in $51 \%$ of patients.

A Framingham risk score higher than $12(10 \%$ mortality in 10 years) was encountered in $23 \%$ of patients and $13 \%$ a score higher than 17 (30\% mortality in 10 years).

The multivariate analysis indicate ferritin $(p=0,03)$ and urea $(p=0,02)$, as independent prediction factors for higher values of the Framingham score; the CXCL-10 serum levels showed a significant correlation with both tests (fig. 4).



Figure 2 - Results of the paired t-test for prediction of the metabolic syndrome 


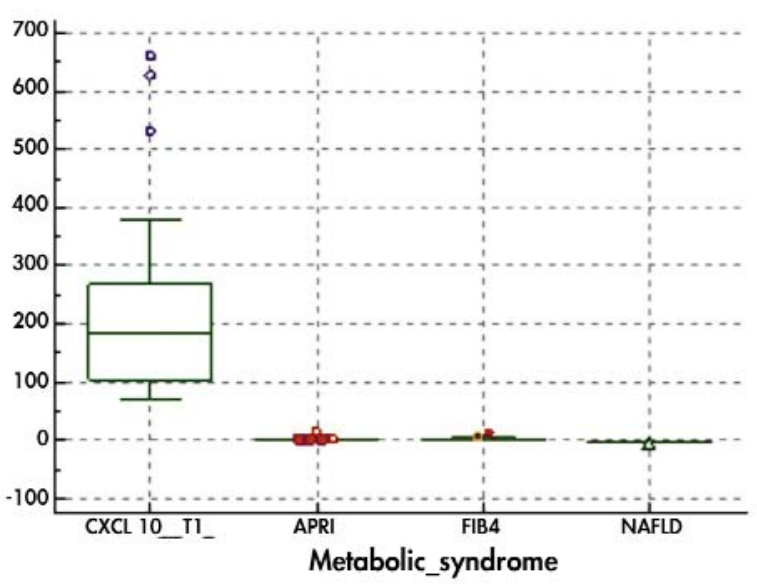

Figure 3 - Comparison of the use of CXCL-10 and non-invasive scores in predicting the presence of the metabolic syndrome

The Spearman rank correlation test showed a significant association between the serum levels of CXCL10 and ferritin $(p=0,04)$, urea $(p=0,003)$, but also with uric acid $(p=0,04)$, an already established risk factor for cardiovascular mortality (table 2).

\section{DISCUSSION}

In our cohort of liver transplant recipients, monitored using routine blood tests to calculate noninvasive score for liver fibrosis and steatosis, and Framingham risk score for cardiovascular complications, we demonstrate a correlation between CXCL-10 and liver fibrosis, steatosis and cardiovascular risk prediction. CXCL10 has been already proposed as a surrogate biomarker for a sustained virological response to interferon-based therapy in patients with chronic hepatitis C $(10,11)$.

An association between the baseline level of CXCL10 and the Framingham risk score originally used to make the decision for initiation of primary prevention of atherosclerosis with statins, could be used to recommend diet and lifestyle modifications for patients with NAFLD (12).

The bi-directional relationship between NAFLD and metabolic syndrome has been suggested by many

Table 2 - Rank correlation of CXCL-10 values

\begin{tabular}{lcc}
\hline Rank correlation & Spearman's coefficient (rho) & P-value \\
\hline Ferritin & 0,364 & 0,04 \\
\hdashline Urea & 0,398 & 0,0035 \\
\hdashline Uric acid & 0,33 & 0,04 \\
\hline
\end{tabular}

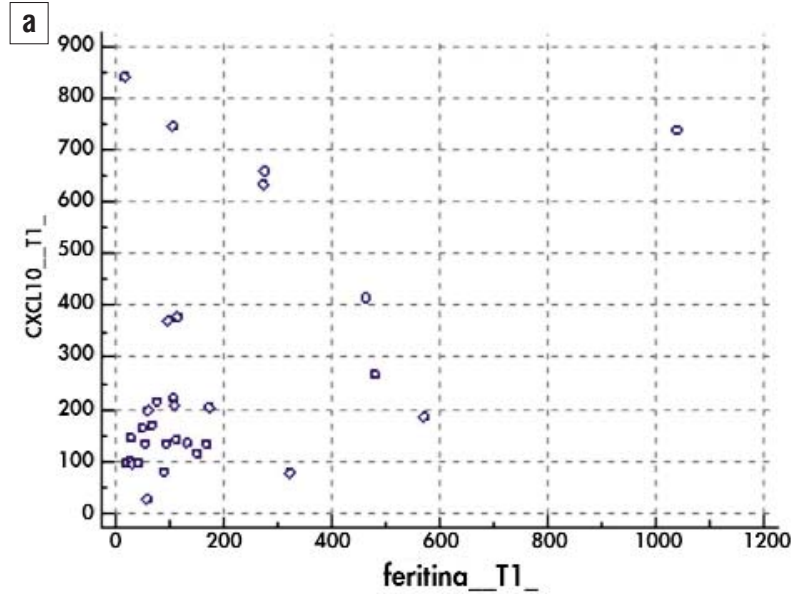

b



Figure 4 - Figure 4: Paired t-test for CXCL-10 and serum ferritin (a) vs serum urea (b)

studies and seems to be confirmed by our recent data. Recent studies related NAFLD as a risk factor for cardiovascular disease (CVD), but even though NAFLD is more prevalent in patients with important cardiovascular risk factors (e.g., T2DM, obesity and hypertension), the relation between NAFLD and CVD seems to be independent of these factors as it strongly influenced by the presence of continuous inflammation, insulin resistance, dyslipidemia, oxidative stress and dysregulations of adipokines.

This type of relationship has been described in insulin resistance, often suggested to be the underlying cause of metabolic syndrome. Insulin resistance can lead to the overproduction of very low-density lipoproteins and accelerated transportation of free fattyacids to liver, which can trigger hepatic steatosis. On the other hand, when hepatic steatosis is present, intermediate products in the process of lipolysis or de novo-lipogenesis such as diacylglycerol may further damage insulin signaling pathways. 
Extrapolating the bi-directional relationship to cardiovascular diseases, and acknowledging the role of NAFLD as an independent risk factor can be a tool for treatment at an early stage and also for avoiding life-threatening complications. Targeting this study in a population of liver transplant recipients shows a different perspective over NAFLD and metabolic syndrome compared to compared to large general populational based studies, as there is an accelerated progression of the metabolic syndrome in a short amount of time after liver transplantation (1-2 years), rapidly reflected by simple blood and imagistic tests.

Serum uric acid was identified as an endogenous danger signal which is upregulated, along with its receptor and multiple signals can result in metabolic insults that lead to the progression of NAFLD to NASH and fibrosis stage (13).

Uric acid seems to be involved in many metabolic features (14) through several mechanisms, including impairing endothelial function, activating both the renal and intracellular renin angiotensin system and inducing intracellular oxidative stress via activation of both in the cytosol and mitochondria NADPH oxidases (15). Some of the same mechanism have been cited also in the influence of BUN over the metabolic syndrome, though with less clinical evidence (16).

Hyperferritinemia, which is considered to be an independent predictor of cardiovascular damage is attributed to the presence of inflammation, metabolic alterations, and hepatocytes necrosis. Serum level of ferritin and transferrin have been proposed as potential surrogate markers for progressive hepatic parenchymal disease in chronic hepatitis (17).

Given the intricate relationship of both CXCL-10 serum values and the results of the Framingham risk score toward uric acid, serum ferritin and serum urea we can state an influence of the serum levels of CXCL-10 over the cardiovascular risk in patients with metabolic syndrome. Further longitudinal studies are necessary in order to confirm the prognostic value of biomarkers, including CXCL10 in cardiovascular related mortality in these patients.

\section{CONCLUSION}

Serum levels of CXCL10 could have an important role in assessing the cardiovascular risk in liver transplant recipients, especially when features of the metabolic syndrome are present.

As cardiovascular complications are the main risk for mortality in patients with NAFLD/NASH, obtaining an early stage diagnosis, and a dynamic evaluation using blood based biomarkers may be the a major improvement for health and lifestyle in these patients.

\section{Conflict of interest}

\author{
All author declare that they have no conflict of \\ interest.
}

\section{REFERENCES}

1. Chalasani N, Younossi Z, Lavine JE, Diehl AM, Brunt EM, Cusi K, et al. The diagnosis and management of non-alcoholic fatty liver disease: practice Guideline by the American Association for the Study of Liver Diseases, American College of Gastroenterology, and the American Gastroenterological Association. Hepatology. 2012; 55(6):2005-23.

2. Wójcik-Cichy K, Koslinska-Berkan E, Piekarska A. The influence of NAFLD on the risk of atherosclerosis and cardiovascular diseases. Clin Exp Hepatol. 2018;4(1):1-6.

3. Bosomworth NJ. Practical use of the Framingham risk score in primary prevention: Canadian perspective. Can Fam Physician. 2011;57(4):417-23.

4. Byrne CD, Targher G. NAFLD: a multisystem disease. J Hepatol. 2015;62(1 Suppl):S47-64.

5. Crisan D, Grigorescu MD, Radu C, Suciu A, Grigorescu M. Interferon- $\gamma$-inducible protein-10 in chronic hepatitis C: Correlations with insulin resistance, histological features \& sustained virological response. Indian J Med Res. 2017;145(4):543-550. doi: 10.4103/ijmr.IJMR_1410_14.

6. Lin ZH, Xin YN, Dong QJ, Wang Q, Jiang XJ, Zhan SH, et al. Performance of the aspartate aminotransferase-to-platelet ratio index for the staging of hepatitis C-related fibrosis: an updated metaanalysis. Hepatology. 2011;53(3):726-36.

7. Bhat M, Rollet-Kurhajec KC, Bhat A, Farag A, Deschenes M, Wong P, et al. Incidence and Predictors of Advanced Liver Fibrosis by a Validated Serum Biomarker in Liver Transplant Recipients. Can J Gastroenterol Hepatol. 2017;2017:4381864.

8. Karlas T, Kollmeier J, Böhm S, Müller J, Kovacs P, Tröltzsch M, et al. Noninvasive characterization of graft steatosis after liver transplantation. Scand J Gastroenterol. 2015; 50(2):224-32.

9. Sánchez-Jiménez BA, Brizuela-Alcántara D, Ramos-Ostos MH, Alva-López LF, Uribe-Esquivel M, Chávez-Tapia NC. Both alcoholic and non-alcoholic steatohepatitis association with cardiovascular risk and liver fibrosis. Alcohol. 2018;69:63-67.

10. Sultana C, Oprisan G, Grancea C, Ruta S. Interferon-gamma Inducible Protein 10-biomarker for treatment outcome in chronic hepatitis C. Rom Biotechnol Lett. 2017;22(6):13155-62.

11. Wang J, Jiang D, Rao H, Yang R, Wang Y, Wei L. Association of interferon- $\gamma$-induced protein-10 serum levels with virological responses to PEG-interferon-based therapy in hepatitis $\mathrm{C}$ virus genotype 1 or 2 chronically infected Chinese patients. Scand J Gastroenterol. 2014;49(11):1349-58.

12. Ballestri S, Lonardo A, Bonapace S, Byrne CD, Loria P, Targher G. Risk of cardiovascular, cardiac and arrhythmic complications in patients with non-alcoholic fatty liver disease. World J Gastroenterol. 2014;20(7):1724-45.

13. Pisano G, Lombardi R, Fracanzani AL. Vascular Damage in Patients with Nonalcoholic Fatty Liver Disease: Possible Role of Iron and Ferritin. Lonardo A, Targher G, eds. Int J Mol Sci. 2016:17(5): 675.

14. Ganz M, Bukong TN, Csak T, Saha B, Park J-K, Ambade A, et al. Progression of non-alcoholic steatosis to steatohepatitis and fibrosis parallels cumulative accumulation of danger signals that promote inflammation and liver tumors in a high fat-cholesterolsugar diet model in mice. J Transl Med. 2015;13:193.

15. Liu X, Zhang H, Liang J. Blood urea nitrogen is elevated in patients with non-alcoholic fatty liver disease. Hepatogastroenterology. 2013; 60(122):343-5.

16. Kanbay M, Jensen T, Solak Y, Le M, Roncal-Jimenez C, Rivard C, et al. Uric acid in metabolic syndrome: From an innocent bystander to a central player. Eur J Intern Med. 2016:29:3-8.

17. Vagu C, Sultana C, Ruta S. Serum Iron Markers in Patients With Chronic Hepatitis C Infection Hepat Mon. 2013;13(10): e13136. 